\title{
Wheat Leaves: The Surface Disposition of Their Epicuticular Wax
}

\author{
E.A. Favret ${ }^{1,2}$ and B. Pidal ${ }^{1}$ \\ ${ }^{1}$ Instituto Nacional de Tecnología Agropecuaria (INTA), CIRN. De Los Reseros y N. Repetto s/n. \\ 1686 Hurlingham. Buenos Aires. Argentina. \\ ${ }^{2}$ CONICET. Av. Rivadavia 1917. C1033AAJ. Ciudad de Buenos Aires. Argentina
}

It is known that RIMAPS (Rotated Image with Maximum Average Power Spectrum) technique allows finding the main directions of any surface topography [1]. During the last years it has been applied to the study of metallic surfaces and biological samples [1,2]. This research work describes the micro-nano-patterning of the epicuticular wax of Triticum aestivum (wheat) leaf surface (adaxial side), by analyzing their RIMAPS spectra. It is known that the epicuticular wax of the wheat leaf has a platelet shape. The basic idea of this work is to see if the platelets have a specific arrangement on the surface, if they are disposed in certain directions. This concept is important because some studies concerning wheat leaf-Rust fungus interaction which were performed to investigate factors influencing fungus spore germination and growing, like leaf surface morphology and chemical components, showed that topological features and wax related components play a crucial role in the orientation of rust germination hyphae towards the stomata, probably modulated by chemical signals [3]. It was observed that hyphae showed a tendency of growing perpendicular to the cell boundaries on wheat leaves. The epicuticular wax of a durable resistant variety to this disease and one susceptible are analyzed. Dried samples from herbarium are chosen and coated with gold for their observation with the scanning electron microscope (SEM, Quanta 200). Figures 1 and 2 show the wheat leaf of resistant and susceptible varieties, respectively, at low magnification (180x). Small and large prickles and leaf veins, parallel to the apex direction $\left(90^{\circ}\right)$, are distinguished. Epidermal cells as well as stomata are more difficult to observe because of the surface shrinkage (it is a herbarium sample). Figures 3 and 4 show the epicuticular wax found on those wheat varieties, respectively (magnification 30000x). The platelet shape of the wax is clearly seen. Figure 5 shows the RIMAPS spectra of figures 3 and 4. Both spectra seem to be quite similar, indicating four big blocks with maxima after a complete rotation. The arrangement of the platelets on the surface, which is not modify by the shrinkage of epidermal cells, has two main directions, one around $45^{\circ}$ $\left(225^{\circ}\right)$ and the other around $135^{\circ}\left(315^{\circ}\right)$. Both spectra have a few differences, e.g. the wax pattern of the susceptible variety shows alignment around $0^{\circ}\left(180^{\circ}\right)$, while the wax pattern of the resistant variety has one explicit alignment around $70^{\circ}\left(250^{\circ}\right)$. Figure 6 shows the average of the RIMAPS spectra of figure 3 and 4 for a better visualization of the spectrum. This is the fingerprint spectrum for the epicuticular wax of wheat leaves. In conclusion, the disposition of the epicuticular wax is not chaotic, it has a precise angular distribution pattern. The main direction of the platelets is just at $45^{\circ}$ at both sides from the apex. RIMAPS spectrum is a useful tool for determining the main characteristics of the micro-nano-patterning.

\section{References}

[1] N.O. Fuentes and E.A. Favret, Journal of Microscopy. 206 (2002) 72-83.

[2] E.A. Favret and B. Pidal. Microscopy and Microanalysis. 17 (Suppl 2) (2011) 344-345.

[3] R.C. Staples et al. Canadian Journal of Plant Pathology. 7 (3) (1985) 314-322.

[4] The authors gratefully acknowledge Adriana Domínguez and Pablo Peitsch (CNEA). 


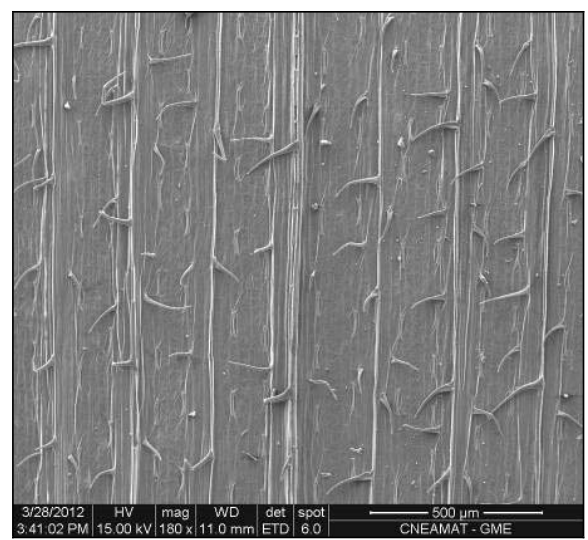

Figure 1. SEM image of the wheat leaf surface (resistant variety)

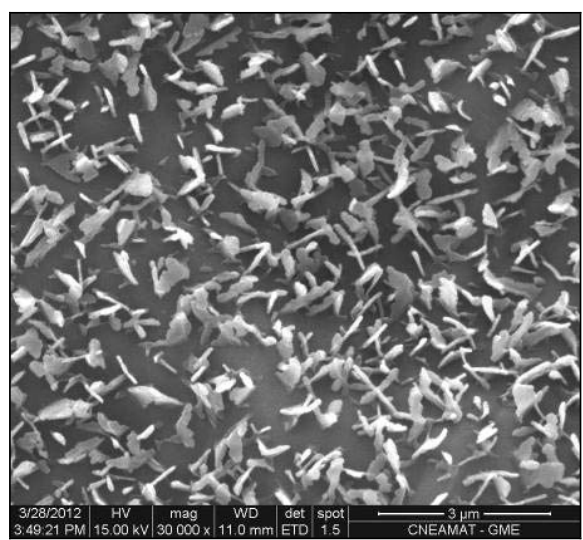

Figure 3. SEM image of the epicuticular wax (resistant variety)

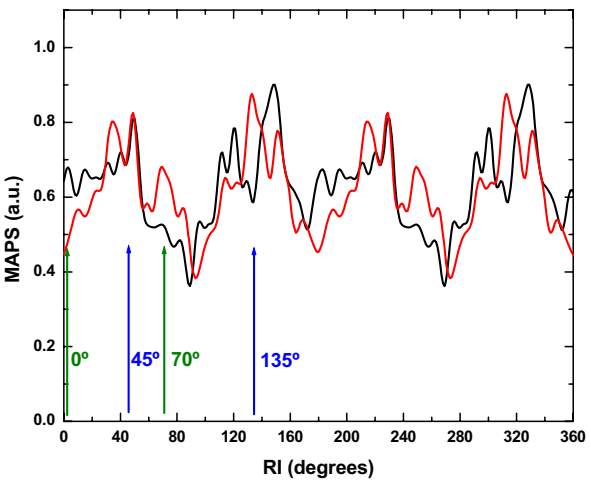

Figure 5. RIMAPS spectra of figs. 3 (red line) and 4 (black line)

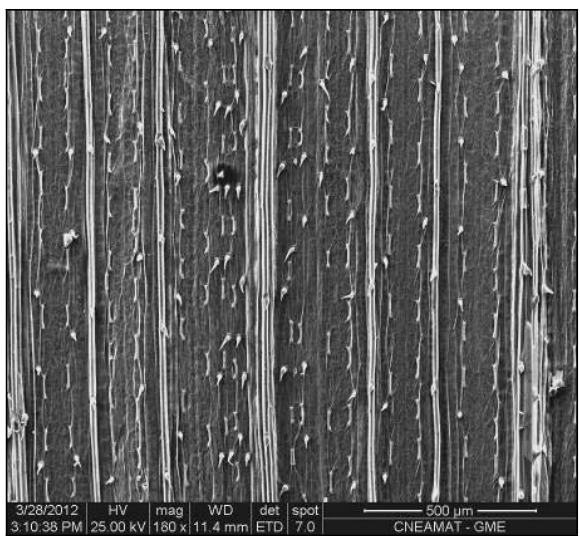

Figure 2. SEM image of the wheat leaf surface (susceptible variety)

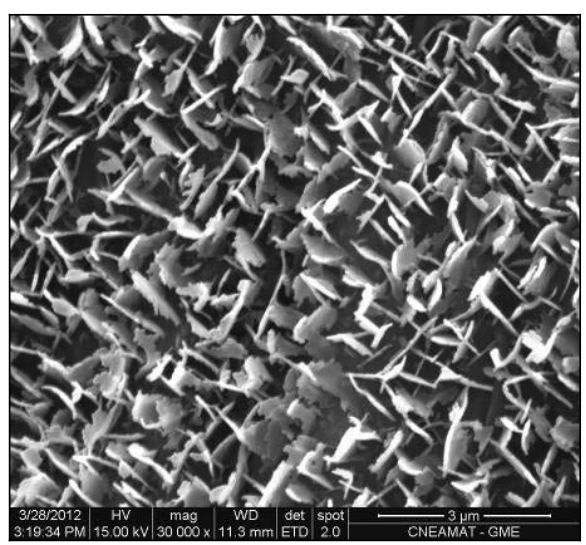

Figure 4. SEM image of the epicuticular wax (susceptible variety)

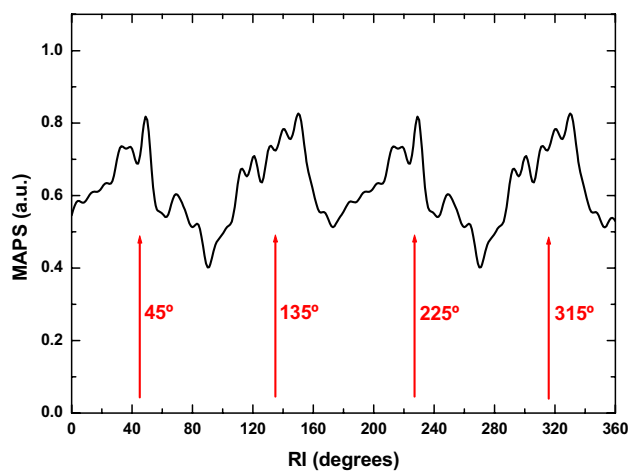

Figure 6: Average RIMAPS spectrum of figure 5 